\title{
On stability regions of the modified midpoint method for a linear delay differential equation
}

\author{
Jana Hrabalová* and Petr Tomášek
}

"Correspondence:

yhraba05@stud.fme.vutbr.cz Institute of Mathematics, Brno University of Technology, Technická

2, Brno, 616 69, Czech Republic

\begin{abstract}
The paper deals with stability regions of a certain discretization of a linear differential equation with constant delay. The main aim of the paper is to analyze the regions of asymptotic stability of the modified midpoint method applied to a linear differential equation with constant delay. Obtained results are compared with other known results, particularly for Euler discretization. The relation between asymptotic stability conditions in the discrete case and continuous case is discussed, too.
\end{abstract}

\section{Introduction}

Qualitative investigation of difference equations plays a key role in the numerical analysis of differential equations. Particularly, the study of asymptotic stability of numerical schemes (including construction of stability regions) is based on the results for asymptotic stability of difference equations. In this paper we deal with the necessary and sufficient asymptotic stability conditions for a certain discretization applied to the linear delay differential equation

$$
y^{\prime}(t)=a y(t)+b y(t-\tau), \quad t>0,
$$

where $a, b, \tau \in \mathbb{R}, \tau>0$. In particular, we compare the obtained conditions with results known for another numerical discretization applied to (1) and also with asymptotic stability conditions for delay differential equation (1) itself. For this purpose, we mention several works, which deal with the asymptotic properties of (1) and some of its discrete counterparts. First, we recall the papers of Andronov and Mayer [1], and of Hayes [2], where the necessary and sufficient conditions for asymptotic stability of (1) were derived. These conditions can be captured as follows:

$$
a \leq b<-a \text { for all } \tau>0
$$

and, in addition to the previous,

$$
|a|+b<0 \quad \text { for } \tau<\frac{\arccos (-a / b)}{\left(b^{2}-a^{2}\right)^{1 / 2}} .
$$

@ 2013 Hrabalová and Tomášek; licensee Springer. This is an Open Access article distributed under the terms of the Creative Commons Attribution License (http://creativecommons.org/licenses/by/2.0), which permits unrestricted use, distribution, and reproduction in any medium, provided the original work is properly cited. 
Particularly, considering $a=0$, equation (1) turns to

$$
y^{\prime}(t)=b y(t-\tau), \quad t>0
$$

and it is asymptotically stable if and only if $-\pi / 2<b \tau<0$.

The first result for a discrete case (related to (3)) that we mention is the paper of Levin and May [3], where the difference equation

$$
y(n+1)-y(n)+\beta y(n-\ell)=0, \quad n=0,1,2, \ldots,
$$

$\ell \geq 1, \ell \in \mathbb{Z}$ was investigated. The necessary and sufficient condition for asymptotic stability of (4) is $0<\beta<2 \cos (\ell \pi /(2 \ell+1))$. A more general case of (4) in the form

$$
y(n+1)+\alpha y(n)+\beta y(n-\ell)=0, \quad n=0,1,2, \ldots,
$$

$\ell \geq 1, \ell \in \mathbb{Z}$ was discussed by Kuruklis [4].

Theorem 1 Let $\alpha \neq 0, \beta$ be arbitrary reals. Equation (5) is asymptotically stable if and only if $|\alpha|<(\ell+1) / \ell$, and

$$
\begin{aligned}
& |\alpha|-1<\beta<\left(\alpha^{2}+1-2|\alpha| \cos \phi\right)^{1 / 2} \quad \text { for } \ell \text { odd, } \\
& |\alpha+\beta|<1 \text { and }|\beta|<\left(\alpha^{2}+1-2|\alpha| \cos \phi\right)^{1 / 2} \quad \text { for } \ell \text { even, }
\end{aligned}
$$

where $\phi \in(0, \pi /(\ell+1))$ is a solution of the auxiliary equation $\sin (\ell x) / \sin ((\ell+1) x)=1 /|\alpha|$.

We note that for $\alpha=0$ the necessary and sufficient condition for asymptotic stability of (5) becomes $|\beta|<1$. We remark that the conditions in this famous result have an implicit form with respect to $\ell$. Another equivalent set of conditions in an explicit form with respect to $\ell$ is introduced in [5].

The analysis of this paper is based on the assertion by Čermák and Tomášek [6], which formulates the necessary and sufficient asymptotic stability conditions for the difference equation

$$
y(n+2)+\alpha y(n)+\beta y(n-\ell)=0, \quad n=0,1,2, \ldots,
$$

where $\alpha, \beta \in \mathbb{R}$ and $\ell \geq 1, \ell \in \mathbb{Z}$.

Theorem 2 Let $\alpha, \beta$ be arbitrary reals such that $\alpha \beta \neq 0$.

(i) Let $\ell$ be even and $\beta(-\alpha)^{\ell / 2+1}<0$. Then (6) is asymptotically stable if and only if

$$
|\alpha|+|\beta|<1 .
$$

(ii) Let $\ell$ be even and $\beta(-\alpha)^{\ell / 2+1}>0$. Then (6) is asymptotically stable if and only if either

$$
|\alpha|+|\beta| \leq 1
$$


or

$$
|| \alpha|-| \beta||<1<|\alpha|+|\beta|, \quad \ell<2 \arccos \frac{\alpha^{2}+\beta^{2}-1}{2|\alpha \beta|} / \arccos \frac{\alpha^{2}-\beta^{2}+1}{2|\alpha|}
$$

holds.

(iii) Let $\ell$ be odd and $\alpha<0$. Then (6) is asymptotically stable if and only if (7) holds.

(iv) Let $\ell$ be odd and $\alpha>0$. Then (6) is asymptotically stable if and only if either (8), or

$$
\beta^{2}<1-\alpha<|\beta|, \quad \ell<2 \arcsin \frac{1-\alpha^{2}-\beta^{2}}{2|\alpha \beta|} / \arccos \frac{\alpha^{2}-\beta^{2}+1}{2|\alpha|}
$$

holds.

Recently, Ren [7] also gave an equivalent system of necessary and sufficient conditions for asymptotic stability of (6), but his formulation needs to solve a nonlinear auxiliary equation, similarly to the result of Kuruklis mentioned above. A description of the stability boundary for (6) in terms of some straight lines and certain parametric curves can be found in Kipnis and Nigmatullin [8].

We close the survey of the results for various linear difference equations with Dannan [9], where a general form of a three-term difference equation

$$
y(n+m)+\alpha y(n)+\beta y(n-\ell)=0, \quad n=0,1,2, \ldots,
$$

with positive integers $m, \ell$ was investigated.

The above mentioned results can be utilized to describe stability regions (i.e., sets of pairs $(a, b) \in \mathbb{R} \times \mathbb{R}$, for which the given discretization is asymptotically stable considering given stepsize) for various numerical schemes, which solve an initial value problem for (1). For more details about numerical background, methods and their stability theory, see, e.g., Bellen and Zennaro [10] and in' t Hout [11].

The paper is focused on the asymptotic properties of a numerical scheme, which arises from (1) by use of the modified midpoint method. The numerical formula is derived by integration over two steps, where the integrals of terms on the right-hand side of (1) are approximated via the trapezoidal rule and the midpoint rule, respectively. The analysis is realized on the equidistant mesh $t_{n}=n h, n=0,1, \ldots$ with stepsize $h=\tau / k$, where $k \geq 2$ is a positive integer. Such an efficient choice of stepsize makes the discretization formulae free of extra interpolation terms, which can arise from an appropriate approximation of the delayed term (see [10]). First, we apply the modified midpoint method to equation (1) to obtain a linear difference equation

$$
Y(n+2)-\frac{1+a h}{1-a h} Y(n)-\frac{2 b h}{1-a h} Y(n-k+1)=0, \quad n=0,1, \ldots
$$

where the stepsize $h$ satisfies $a h \neq 1$. The value $Y(n)$ then represents a numerical approximation of solution $y$ of delay differential equation (1) at the nodal point $t_{n}$.

The paper is organized as follows. Section 2 presents the set of necessary and sufficient conditions for asymptotic stability of (11). In Section 3 we discuss some important properties of obtained results and compare them with the results known for another discretization as well as with the asymptotic stability conditions for the corresponding differential equation. Section 4 concludes the paper by final remarks. 


\section{Main result}

In this section we state the necessary and sufficient conditions for asymptotic stability of (11). The analysis of (11) falls naturally into two parts according to the parity of $k$. For an effective and clear formulation of the main result, we introduce the symbols

$$
\begin{gathered}
\tau_{1}^{*}(h)=h+2 h \arcsin \frac{a+b^{2} h}{(1+a h)|b|} / \arccos \frac{1+a^{2} h^{2}-2 b^{2} h^{2}}{a^{2} h^{2}-1}, \\
\tau_{2}^{*}(h)=h+2 h \arccos \frac{a+b^{2} h}{|(1+a h) b|} / \arccos \frac{1+a^{2} h^{2}-2 b^{2} h^{2}}{\left|a^{2} h^{2}-1\right|},
\end{gathered}
$$

which are utilized in these two parts, respectively.

Theorem 3 (I) Let $k \geq 2$ be even. Then (11) is asymptotically stable if and only if one of the following conditions holds:

$$
\begin{aligned}
& |b h| \leq 1, \quad|b|+a<0, \\
& 2<2 b^{2} h^{2}<1-a h, \quad \tau<\tau_{1}^{\prime \prime}(h) .
\end{aligned}
$$

(II) Let $k \geq 3$ be odd and $m=(k-1) / 2$. Then (11) is asymptotically stable if and only if one of the following conditions holds:

$$
\begin{aligned}
& a \leq b<-a, \quad|b h|<1, \\
& |b|+a<0, \quad(-1)^{m} b h=1, \\
& b+|a|<0, \quad b h>-1, \quad \tau<\tau_{2}^{*}(h), \\
& (-1)^{m} b+a<0, \quad(-1)^{m} b h>1, \quad \tau<\tau_{2}^{\prime \prime}(h), \\
& (-1)^{m} b+a>0, \quad(-1)^{m+1} b h>1, \quad \tau<\tau_{2}^{*}(h) .
\end{aligned}
$$

Proof The proof is based on the application of Theorem 2 to (11) and the ensuing analysis of the obtained conditions. In particular, if we consider (11) in the form of (6), the coefficients $\alpha$ and $\beta$ of (6) are given by

$$
\alpha=-\frac{1+a h}{1-a h}, \quad \beta=-\frac{2 b h}{1-a h}
$$

and the indices $\ell$ and $k$ are in the relation $\ell=k-1$.

Case (I): Investigating the case of $k$ even, we utilize parts (iii) and (iv) of Theorem 2. Firstly, we focus on condition (iii): considering the coefficients (19), the assumption $\alpha<0$ implies $|a h|<1$. Thus, 7 is equivalent to $|b|+a<0$. Therefore, condition (iii) coincides with $|b h|<-a h<1$.

Now, we analyze condition (iv): analogously, assumption $\alpha>0$ implies $|a h|>1$. Hence, (8) gives $|b h| \leq 1$ providing $a h<-1$, while for the case $a h>1$ relation (8) cannot occur. We now turn to (10). Relation $(10)_{1}$ can be read as $2<2 b^{2} h^{2}<1-a h$. Furthermore, the restriction $(10)_{2}$ becomes

$$
k-1<2 \arcsin \frac{a+b^{2} h}{|(1+a h) b|} / \arccos \frac{1+a^{2} h^{2}-2 b^{2} h^{2}}{\left|a^{2} h^{2}-1\right|} .
$$


Since $|a h|>1$ and $k=\tau / h$, it can be written as $\tau<\tau_{1}^{*}(h)$. Therefore, condition (iv) is satisfied if and only if either $|b h| \leq 1, a h<-1$ or (13).

Finally, Theorem 2 does not cover the case of $\alpha \beta=0$ (i.e., $a h=-1$ or $b=0$ ). In our case we do not consider the eventuality $b=0$ with respect to the fact that we deal with the discretization of (1). Accordingly, for $a h=-1$, equation (11) turns to

$$
Y(n+1)-b h Y(n-k+1)=0, \quad n=0,1, \ldots
$$

and the necessary and sufficient condition for its asymptotic stability is given by Theorem 1 as $|b h|<1$. Summarizing the above discussion, we conclude that if $k$ is even, (11) is asymptotically stable if either (12) or (13) holds.

Case (II): For $k$ odd, we consider conditions (i) and (ii) of Theorem 2. Condition (i) can be rewritten as

$$
\frac{-2 b h}{1-a h}\left(\frac{1+a h}{1-a h}\right)^{1+m}<0, \quad\left|\frac{1+a h}{1-a h}\right|+\left|\frac{2 b h}{1-a h}\right|<1
$$

by use of (19). With respect to the parity of power in the first relation, we obtain by a sign discussion of terms in the other relation a set of conditions equivalent to (20) as

$$
\begin{array}{lll}
|a h|<1, & b>0, & a<-b, \\
a h<-1, & b>0, & b h<1
\end{array}
$$

for $m$ odd and (21),

$$
a h<-1, \quad b<0, \quad-b h<1
$$

for $m$ even.

In the Case (ii) of Theorem 2, condition (8) can be reformulated as

$$
\frac{-2 b h}{1-a h}\left(\frac{1+a h}{1-a h}\right)^{1+m}>0, \quad\left|\frac{1+a h}{1-a h}\right|+\left|\frac{2 b h}{1-a h}\right| \leq 1 .
$$

An analogous analysis to that above shows that for $m$ odd, (22) is equivalent to

$$
\begin{aligned}
& |a h|<1, \quad b<0, \quad a \leq b, \\
& a h<-1, \quad b<0, \quad-b h \leq 1 .
\end{aligned}
$$

In the case $m$ even, condition (22) is satisfied if and only if (23) or

$$
a h<-1, \quad b>0, \quad b h \leq 1
$$

holds. The above discussion of the Case (i), the part of (ii) considering (8) and including the case $\alpha=0$ (i.e., $a h=-1,|b h|<0$, see Case (I)) gives (14)-(15).

Now it remains to analyze condition (9) adapted for equation (11) by (19), i.e.,

$$
|| \frac{1+a h}{1-a h}|-| \frac{2 b h}{1-a h}||<1<\left|\frac{1+a h}{1-a h}\right|+\left|\frac{2 b h}{1-a h}\right|, \quad \tau<\tau_{2}^{*}(h)
$$


under the assumption $\frac{-2 b h}{1-a h}\left(\frac{1+a h}{1-a h}\right)^{1+m}>0$. In the same manner as above, we get the equivalency to the following set of conditions:

$$
\begin{aligned}
& |a h|<1, \quad b<0, \quad 1+a h \leq-2 b h, \quad-b h<1, \quad b<a, \quad \tau<\tau_{2}^{*}(h), \\
& |a h|<1, \quad b<0, \quad 1+a h>-2 b h, \quad b<-|a|, \quad \tau<\tau_{2}^{*}(h), \\
& a h>1, \quad b>0, \quad 1+a h \leq 2 b h, \quad b<a, \quad \tau<\tau_{2}^{*}(h), \\
& a h>1, \quad b>0, \quad 1+a h>2 b h, \quad 1<b h, \quad \tau<\tau_{2}^{*}(h), \\
& a h<-1, \quad b<0, \quad 1+a h<2 b h, \quad-b h>1, \quad \tau<\tau_{2}^{*}(h), \\
& a h<-1, \quad b<0, \quad 1+a h \geq 2 b h, \quad-b h>1, \quad a<b, \quad \tau<\tau_{2}^{*}(h)
\end{aligned}
$$

for $m$ odd and (24), (25),

$$
\begin{aligned}
& a h>1, \quad b<0, \quad 1+a h \leq-2 b h, \quad-b<a, \quad \tau<\tau_{2}^{*}(h), \\
& a h>1, \quad b<0, \quad 1+a h>-2 b h, \quad 1<-b h, \quad \tau<\tau_{2}^{\prime \prime}(h), \\
& a h<-1, \quad b>0, \quad 1+a h<-2 b h, \quad b h>1, \quad \tau<\tau_{2}^{*}(h), \\
& a h<-1, \quad b>0, \quad 1+a h \geq-2 b h, \quad b h>1, \quad a<-b, \quad \tau<\tau_{2}^{*}(h)
\end{aligned}
$$

for $m$ even. These conditions are jointly expressed by (16)-(18). In fact, (16) coincides with (24), (25). Condition (17) is equivalent to (28), (29) and (32), (33) for $m$ odd and $m$ even, respectively. Finally, (18) is the same as (26), (27) for $m$ odd and (30), (31) for $m$ even. The proof is complete.

\section{Asymptotic stability discussion}

In this section we introduce some remarks and comments to the obtained result formulated in Theorem 3. First, we focus on the connection with the asymptotic stability properties of (1). Particularly, we investigate a limit form of Theorem 3 considering $h \rightarrow 0$. In the Case (I) of $k$ even, the asymptotic stability region of (11) becomes $|b|+a<0$. Let us note that with the exception of the boundary, this region corresponds to (2). In the Case (II) of $k$ odd, it may be shown (by the L'Hospital rule) that the asymptotic stability conditions turn to

$$
\begin{aligned}
& a \leq b<-a, \\
& |a|+b<0, \quad \tau<\frac{\arccos (-a / b)}{\left(b^{2}-a^{2}\right)^{1 / 2}}
\end{aligned}
$$

as $h \rightarrow 0$. These are equivalent to the conditions defining the asymptotic stability region of (1).

Now we present the necessary and sufficient conditions for the asymptotic stability of the midpoint method discretization of (3) in the form

$$
Y(n+2)-Y(n)-2 b h Y(n-k+1)=0, \quad n=0,1, \ldots
$$

and then we focus on some of their monotony properties with respect to changing stepsize $h$. Finally, we compare the obtained stability intervals with the stability interval of the 
corresponding differential equation, as well as with the stability intervals for the forward Euler method discretization of (3).

Corollary 4 Equation (34) is asymptotically stable if and only if

$$
\text { kis odd, } \quad 0>b>-\frac{1}{h} \sin \frac{\pi h}{2 \tau} .
$$

Proof The assertion is an immediate consequence of Theorem 3. Setting $a=0$, we realize that conditions (12) and (13) cannot occur. Therefore, (34) is unstable for any $b \in \mathbb{R}$ in the case of $k$ even.

Considering $k$ odd, we investigate conditions (14)-(18). For $a=0$, there arises a contradiction in all conditions except for (16), which becomes

$$
b<0, \quad b h>-1, \quad \tau<h+\frac{2 h \arccos (-b h)}{\arccos \left(1-2 b^{2} h^{2}\right)} .
$$

We omit (35) 2 because it is imposed by the domain of the last relation $(35)_{3}$. Our next aim is to simplify the delay restriction $(35)_{3}$ to the form more convenient for further analysis. We use the formula

$$
\arccos \left(1-2 x^{2}\right)=\pi-2 \arccos x, \quad x>0
$$

and taking into account that function $\cos x$ is decreasing for $x \in(0, \pi / 2)$, we arrive at

$$
\cos \frac{\pi(\tau-h)}{2 \tau}>-b h
$$

Since $\cos (\pi / 2-x)=\sin x$, we get

$$
\sin \frac{\pi h}{2 \tau}>-b h
$$

Finally, we rewrite (35) as

$$
0>b>-\frac{1}{h} \sin \frac{\pi h}{2 \tau},
$$

which is the necessary and sufficient condition for asymptotic stability of (34) providing $k$ is odd.

We emphasize that the stability regions are captured just by stability intervals for values of parameter $b$. We denote $I_{\tau}^{M}(h)=\left(-\frac{1}{h} \sin \frac{\pi h}{2 \tau}, 0\right)$ stability intervals of (34) derived in Corollary 4. Next assertion describes the relation between stability intervals $I_{\tau}^{M}(h)$ with respect to stepsize $h$.

Theorem 5 Let $3 \leq k_{1}<k_{2}$ be arbitrary positive odd integers and let $h_{1}=\tau / k_{1}>\tau / k_{2}=h_{2}$ be corresponding stepsizes. Then

$$
I_{\tau}^{M}\left(h_{2}\right) \supset I_{\tau}^{M}\left(h_{1}\right) .
$$


Proof Let us define a function

$$
f(h)=-\frac{1}{h} \sin \frac{\pi h}{2 \tau}, \quad h \in(0, \tau / 3\rangle,
$$

which represents the dependence of the left endpoint of $I_{\tau}^{M}(h)$ on stepsize $h$. Since the right endpoint is zero for any $h$, we discuss only the monotony of $f(h)$. Doing this, we drop the constraint $h=\tau / k$ and we consider $f(h)$ as a function of a continuous argument $h$. Then

$$
f^{\prime}(h)=\frac{1}{h^{2}} \sin \frac{\pi h}{2 \tau}-\frac{\pi}{2 \tau h} \cos \frac{\pi h}{2 \tau}, \quad h \in(0, \tau / 3\rangle .
$$

Since $\sin \frac{\pi h}{2 \tau}>0$ for $h \in(0, \tau / 3\rangle, f^{\prime}(h)>0$ when

$$
\cot \frac{\pi h}{2 \tau}<\frac{2 \tau}{\pi h}, \quad h \in(0, \tau / 3\rangle .
$$

If we substitute $x=\frac{\pi h}{2 \tau}$, the last relation becomes

$$
\tan x>x, \quad x \in(0, \pi / 6) .
$$

Obviously, $\tan (0)=0$ and $(\tan (x))^{\prime}=\cos ^{-2}(x)>1=x^{\prime}$ for $x \in(0, \pi / 6\rangle$. Therefore (36) holds for any $x \in(0, \pi / 6\rangle$. Thus, we have proved that $f^{\prime}(h)>0$ for $h \in(0, \tau / 3\rangle$ and consequently $I_{\tau}^{M}\left(h_{2}\right) \supset I_{\tau}^{M}\left(h_{1}\right)$.

Next, we compare stability intervals $I_{\tau}^{M}(h)$ with the stability interval of (3), which we denote $I_{\tau}^{*}=(-\pi /(2 \tau), 0)$.

Remark 6 An important property is the behavior of $I_{\tau}^{M}(h)$ as $h \rightarrow 0$. Using the L'Hospital rule, we may see that

$$
\lim _{h \rightarrow 0}-\frac{1}{h} \sin \frac{\pi h}{2 \tau}=\lim _{h \rightarrow 0}-\frac{\pi}{2 \tau} \cos \frac{\pi h}{2 \tau}=-\frac{\pi}{2 \tau} .
$$

Therefore, $I_{\tau}^{M}(h)$ is approaching $I_{\tau}^{*}$ as $h \rightarrow 0$.

Remark 7 In the proof of Theorem 5 we have shown that $-\frac{1}{h} \sin \frac{\pi h}{2 \tau}$ is an increasing function on $h \in(0, \tau / 3)$. Considering also Remark 6, we conclude that $I_{\tau}^{*} \supset I_{\tau}^{M}(h)$ for any $h=\tau / k$, where $k$ is odd. Note that the midpoint method discretization of (3) is not asymptotically stable.

Finally, we discuss a relation between $I_{\tau}^{M}(h)$ and asymptotic stability intervals for the forward Euler discretization of (3). They are derived in [12], and we denote them as $I_{\tau}^{E}(h)=$ $\left(-\frac{2}{h} \cos \frac{\pi \tau}{2 \tau+h}, 0\right)$.

Theorem 8 Let $k \geq 3$ be an arbitrary positive odd integer and let $h=\tau / k$ be the corresponding stepsize. Then

$$
I_{\tau}^{M}(h) \supset I_{\tau}^{E}(h) .
$$


Proof Since the right endpoints of $I_{\tau}^{M}(h)$ and $I_{\tau}^{E}(h)$ are zero for any $h$, we investigate only the behavior of the left endpoints with respect to changing stepsize $h$. We define a function

$$
g(h)=-\frac{2}{h} \cos \frac{\pi \tau}{2 \tau+h}, \quad h \in(0, \tau),
$$

which expresses the dependence of the left endpoint of $I_{\tau}^{E}(h)$ on $h$. In the further analysis, we drop the constraint $h=\tau / k$ and consider both functions $f(h)$ and $g(h)$ to be functions with a continuous argument for $h \in(0, \tau)$ (we extend the domain of $f(h)$ to simplify the proof). Thus our aim is to show that $f(h)-g(h)<0$ for any $h \in(0, \tau)$, i.e.,

$$
-\sin \frac{\pi h}{2 \tau}+2 \cos \frac{\pi \tau}{2 \tau+h}<0, \quad h \in(0, \tau) .
$$

To do this, we introduce the following proposition.

Lemma Let $F \in C^{3}\langle a, b\rangle$ be a function such that $F(a)=F(b)=0, F^{\prime}(a) \leq 0, F^{\prime}(b)>0$, $F^{\prime \prime}(a)<0, F^{\prime \prime}(b)>0$ and $F^{\prime \prime \prime}(t)>0$ for all $t \in(a, b)$. Then $F(t)<0$ for all $t \in(a, b)$.

Proof Since $F^{\prime \prime \prime}(t)>0$ for all $t \in(a, b)$, the function $F^{\prime \prime}(t)$ is increasing. Since $F^{\prime \prime}(a)<0<$ $F^{\prime \prime}(b)$, there is a unique point $t_{1} \in(a, b)$ such that $F^{\prime \prime}\left(t_{1}\right)=0$. Thus, the function $F^{\prime}(t)$ is decreasing in $\left(a, t_{1}\right)$ and increasing in $\left(t_{1}, b\right)$. Further, since $F^{\prime}\left(t_{1}\right)<F^{\prime}(a) \leq 0$ and $F^{\prime}(b)>0$, there is a unique point $t_{2} \in(a, b)$ such that $F^{\prime}\left(t_{2}\right)=0$. Therefore, $F(t)$ is decreasing in $\left(a, t_{2}\right)$ and increasing in $\left(t_{2}, b\right)$. Taking into account $F(a)=F(b)=0$, we obtain that $F(t)<0$ for $t \in(a, b)$.

Next, we denote $s=2+h / \tau$. Then we define

$$
G(s)=\sin \frac{\pi s}{2}+2 \cos \frac{\pi}{s}, \quad s \in(2,3),
$$

which is equivalent to the left-hand side of (37). It holds that $G(2)=G(3)=0, G^{\prime}(2)=0$, $G^{\prime}(3)=\frac{\sqrt{3} \pi}{9}>0, G^{\prime \prime}(2)=-\frac{\pi}{2}<0$ and $G^{\prime \prime}(3)=\frac{\pi^{2}}{4}-\frac{\pi^{2}}{81}-2 \sqrt{3} \frac{\pi}{27}>0$. Further

$$
G^{\prime \prime \prime}(s)=\frac{12 \pi^{2}}{s^{5}} \cos \frac{\pi}{s}-\frac{\pi^{3}}{8} \cos \frac{\pi s}{2}+\frac{2 \pi}{s^{6}}\left(6 s^{2}-\pi^{2}\right) \sin \frac{\pi}{s}>0,
$$

since each term in the sum is positive for all $s \in(2,3)$. Then by the previous lemma, we have that $G(s)<0$ for all $s \in(2,3)$ and consequently $f(h)<g(h)$ for $h \in(0, \tau)$, which concludes the proof.

\section{Conclusions}

To summarize the previous, the main result formulated in Theorem 3 describes the asymptotic stability regions of difference equation (11). This equation actually represents a discretization of delay differential equation (1) by a modified midpoint rule. It was shown that the asymptotic stability regions depend not only on the value of stepsize $h$, but also on the parity of $k$. In the case $a=0$, the obtained result was given to the connection with the results known for the Euler discretization of (3). Moreover, the connection with asymptotic stability properties of delay differential equation (3) was also mentioned. This discussion points out some interesting properties of the stability regions for the discrete 
form of the delay differential equation (1). The authors believe that analogous investigation is possible also for more complicated numerical formulae (applied to (1)) as far as there are known stability criteria for corresponding difference equations. Such analysis may be done, e.g., for the $\Theta$-method.

Competing interests

The authors declare that they have no competing interests.

\section{Authors' contributions}

The authors declare that the research was realized in collaboration with the same contribution. Both authors read and approved the final manuscript.

\section{Acknowledgements}

The first author was supported by the project FSI-S-11-3 of Brno University of Technology. The second author was supported by the grant P201/11/0768 Qualitative properties of solutions of differential equations and their applications of the Czech Science Foundation.

Received: 1 March 2013 Accepted: 28 May 2013 Published: 18 June 2013

\section{References}

1. Andronov, AA, Mayer, AG: The simplest linear systems with delay. Autom. Remote Control 7(2-3), $95-106$ (1946)

2. Hayes, ND: Roots of the transcendental equations associated with certain difference-differential equation. J. Lond. Math. Soc. 25, 226-232 (1950)

3. Levin, SA, May, R: A note on difference delay equations. Theor. Popul. Biol. 9, 178-187 (1976)

4. Kuruklis, SA: The asymptotic stability of $x_{n+1}-a x_{n}+b x_{n-k}=0$. J. Math. Anal. Appl. 188, 719-731 (1994)

5. Čermák, J, Jánský, J, Kundrát, P: On necessary and sufficient conditions for the asymptotic stability of higher order linear difference equations. J. Differ. Equ. Appl. 18(11), 1781-1800 (2012)

6. Čermák, J, Tomášek, P: On delay-dependent stability conditions for a three-term linear difference equation. Funkc. Ekvacioj (to appear.)

7. Ren, H: Stability analysis of second order delay difference equations. Funkc. Ekvacioj 50, 405-419 (2007)

8. Kipnis, MM, Nigmatullin, RM: Stability of the trinomial linear difference equations with two delays. Autom. Remote Control 65(11), 1710-1723 (2004)

9. Dannan, F: The asymptotic stability of $x(n+k)+a x(n)+b x(n-\ell)=0$. J. Differ. Equ. Appl. 10(6), $589-599$ (2004)

10. Bellen, A, Zennaro, M: Numerical Methods for Delay Differential Equations. Oxford University Press, Oxford (2003)

11. in' $t$ Hout, $\mathrm{KJ}$ : On the stability of adaptations of Runge-Kutta methods to systems of delay differential equations. Appl. Numer. Math. 22, 237-250 (1996)

12. Hrabalová, J: On stability intervals of Euler methods for a delay differential equation. Aplimat - J. Appl. Math. 5(2), 77-84 (2012)

doi:10.1186/1687-1847-2013-177

Cite this article as: Hrabalová and Tomášek: On stability regions of the modified midpoint method for a linear delay differential equation. Advances in Difference Equations 2013 2013:177.

\section{Submit your manuscript to a SpringerOpen ${ }^{\circ}$ journal and benefit from:}

- Convenient online submission

Rigorous peer review

Immediate publication on acceptance

- Open access: articles freely available online

- High visibility within the field

- Retaining the copyright to your article 